\title{
Approach to recurrent CMV disease and antiviral resistance
}

\author{
Quan V. Hoang • David M. Simon • Debra A. Goldstein
}

Received: 28 April 2010 /Accepted: 2 May 2010/Published online: 25 May 2010

(C) Springer-Verlag 2010

\section{Dear Editor,}

We appreciate the opportunity to further discuss our recent report on recurrent cytomegalovirus (CMV) retinitis in a non-HIV patient [1], in particular to respond to the interest and letter from Dr. Wiwanitkit, who asked us to address our approach to recurrent CMV disease and antiviral resistance.

Presently, our patient remains disease-free, without evidence of retinitis or viremia. Favorably, she has not required further immunosuppression for the past 25 months. Treatment with high-dose ( 5,000-6,000 mcg/dose) GCV injections provided adequate local concentrations to inhibit/ control ocular infection. We suspect that lack of recurrent CMV disease is indicative of development of CMV-specific immunity. Our patient, in particular, experienced primary CMV infection with a high viral load during a period of intense immunodeficiency. Systemic antiviral therapy failed to suppress viremia and likely contributed to resistance. Viral clearance paralleled the rise in CD4 T lymphocytes [1]. Although CD4 $\mathrm{T}$ lymphocyte recovery suggests immune reconstitution, CD4 count recovery is not always associated with development of CMV-specific immunity, as demonstrated in an AIDS patient by Johnson et al. [2] and noted by Dr. Wiwanitkit.

Among HIV patients, the best strategy to prevent CMV disease is early HAART, which has been associated with a marked decline in the incidence of CMV in this population. CMV drug resistance today primarily impacts solid-organ transplant recipients. We agree with Dr. Wiwanitkit that optimal treatment of drug-resistant CMV disease is limited and new agents are needed. Our case suggests that suprapharmacologic dosing of antivirals can be effective for treatment of retinitis due to drug-resistant CMV.

\section{References}

1. Hoang QV, Simon DM, Kumar GN, Oh F, Goldstein DA (2010) Recurrent CMV retinitis in a non-HIV patient with drug-resistant CMV. Graefes Arch Clin Exp Ophthalmol 248(5):737-740

2. Johnson SC, Benson CA, Johnson DW, Weinberg A (2001) Recurrences of cytomegalovirus retinitis in a human immunodeficiency virus-infected patient, despite potent antiretroviral therapy and apparent immune reconstitution. Clin Infect Dis 32(5):815-819

Q. V. Hoang $\cdot$ D. A. Goldstein $(\bowtie)$

Department of Ophthalmology and Visual Sciences,

University of Illinois at Chicago,

1855 W. Taylor Street (M/C 648),

Chicago, IL 60612, USA

e-mail: debrgold@yahoo.com

D. M. Simon

Section of Infectious Diseases, Rush University Medical Center,

Chicago, IL 60612, USA 